\title{
2D imprinted substrates and 3D electrospun scaffolds revolutionize biomedicine
}

\author{
"Imprinting lithography and electrospinning \\ are due to transform healthcare."
}

First draft submitted: 16 March 2016; Accepted for publication: 22 March 2016; Published online: 13 April 2016

Keywords: cell response $\bullet$ electrospinning $\bullet$ imprinting $\bullet$ nano-bio interface $\bullet$ tissue response $\bullet$ topography

Momentous advances in engineering in the 1980 s and 1990s reduced significantly the manufacturing cost of equipment that can produce nano to low microscale materials. This was a key milestone in the field of biomedicine, as from the early 2000s, we entered the era of nanobiomedicine. Essentially, with very low capital cost, every laboratory in the world could develop cell culture substrates and/or implantable devices with morphological features down to the nanometer scale. The rationale of using such small dimensional features derives from the fact that the extracellular space is comprised of nano- to microscale supramolecular assemblies. Further, cells employ probing filopodia extensions and contractile intracellular machinery to gather topographical, spatial, mechanical and chemical information from the extracellular environment, which consequently determines cell functionality, lineage commitment and fate. Among the various fabrication technologies, lithography constitutes the most popular 2D top-down method, while electrospinning represents the most widely used 3D bottomup method [1-7]. The produced $2 \mathrm{D}$ and $3 \mathrm{D}$ constructs have enabled the study of cell and tissue responses at previously economical and technological prohibiting small scales and the rational design thereof for a specific application/clinical indication. Key to the design tenant is that topographical features and morphological structures should promote cellular polarization and organized extracel- lular matrix assembly, resembling the natural conformation of tissues.

Using photolithography back in 1983, microscale V-shape grooves were created to study human gingival and porcine epithelial cell response [8]. By 1997, we were in a position to fabricate substrates with feature (square) accuracy down to $14 \mathrm{~nm}$ (depth) to study Xenopus neurites outgrowth using electron-beam lithography $[9,10]$. To date, we have successfully imprinted various gratings and geometric shapes along different scales onto thermoplastic polymers to create biologically relevant topographical interfaces. Data obtained from these and other studies clearly illustrate that topographical features are powerful modulators of cell morphology and differential function [11-15]. Further, nano- and microscale arrays have been shown to be potent tools in maintaining the phenotype fidelity of permanently differentiated cells and in differentiating stem cells toward specific lineages $[16,17]$.

Multiple topographic arrays have also been used as high-throughput systems to elucidate the cell-surface interactions and enable identification of improved surfaces for opti$\mathrm{mal} /$ required cell response $[18,19]$. Specifically, topographical features have been shown to directly modulate cell-material interaction and to affect the composition, orientation and conformation of adsorbed extracellular matrix components [20,21]. Further, nanoimprinted materials have been shown to either

\section{Manus Biggs}

Science Foundation Ireland (SFI) Centre for Research in Medical Devices (CÚRAM), Biomedical Sciences Building, National University of Ireland Galway (NUI Galway), Galway, Ireland

\section{Abhay Pandit}

Science Foundation Ireland (SFI) Centre for Research in Medical Devices (CÚRAM), Biomedical Sciences Building, National University of Ireland Galway (NUI Galway), Galway, Ireland

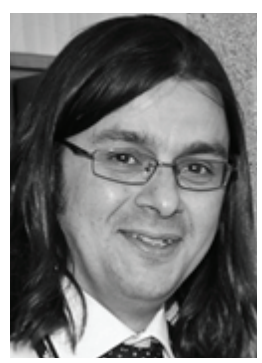

Dimitrios I Zeugolis

Author for correspondence:

Science Foundation Ireland (SFI)

Centre for Research in Medical Devices (CÚRAM), Biomedical Sciences Building, National University of Ireland Galway (NUI Galway), Galway, Ireland and

Regenerative, Modular \& Developmental Engineering Laboratory (REMODEL),

Biomedical Sciences Building, National University of Ireland Galway (NUI Galway), Galway, Ireland

Tel.: +353091493166

Fax: +353091563991

dimitrios.zeugolis@nuigalway.ie 
directly or indirectly influence the formation and maturation of focal adhesion complexes [22], a process though to modulate transcriptional events through adhesiondependent phosphorylation of downstream signaling molecules (e.g., mediated focal adhesion kinases).

Shape memory polymers have also been investigated as responsive nanoscale materials, which can be switched, for example, from a surface, which presents an anisotropic topography to a planar surface with associated loss of cell orientation [23]. Furthermore, analogous systems studying grooves with switchable widths have been employed to apply mechanical force to regulate the shape and the cytoskeletal arrangement of rat stem cells, inducing lineage-specific differentiation of stem cell toward myogenic lineages in the absence of induction factors [24]. It can be inferred that nanoimprinted topographies that display a dynamic response to environmental changes may promote the development of tunable tissue-specific topography and play important future roles in smart, tissue engineered implants or lab-on-chip devices.

Unfortunately, 2D nano to low microimprinted substrates have failed to induce a therapeutic effect in vivo $[25,26]$, which imposes the need for the development of nanotextured 3D scaffold fabrication technologies, such as electrospinning, for implantation purposes. Electrospinning was first appeared in the biomaterials field in 2001 [27,28]. Since then, it has been widely used in every single aspect of biomedicine. Numerous natural and synthetic polymers have been electrospun, although the harsh solvents are associated with denaturation of collagen [29,30]. Even stimuliresponsive polymers have been electrospun for various biomedical applications, including the production of living cell layers [31,32]. Advances in the fabrication process, such as rotating [33-35] or controlled porosity [36-38] collectors have enabled the production of bidirectionally aligned electrospun meshes or meshes with precise architectural features, respectively. Melt electrospinning, a combination of electrospinning and direct writing additive manufacturing, has given rise to a new family of scaffolds with more precise architectural features $[39,40]$. Blending, multilayering, surface treatment, dual electrospinning, coaxial electrospinning and electrospinning-co-electrospraying are a few of the developed technologies that have allowed sustained and localized delivery of bioactive/therapeutics

\section{References}

1 Ryan C, Fuller K, Larranaga A et al. An academic, clinical and industrial update on electro-spun, additive manufactured and imprinted medical devices. Expert Rev. Med. Devices 12(5), 601-612 (2015). molecules at the side of interest [41-43]. Electrospinning-co-electrospraying and coaxial electrospinning have also been used to deposit living cells onto the produced scaffolds [44-47]. With respect to cell response, electrospun mats, alone or in combination with biochemical/biological cues, have been shown to maintain phenotype fidelity of permanently differentiated cells and/or to direct stem cell lineage commitment [48-54]. In preclinical setting, electrospun scaffolds, alone or with bioactive/therapeutic molecules and various viable cell populations have been implanted literally to every single preclinical model known, and the results in all instances have been very promising (301 papers; source: PubMed; term searched: 'electrospinning' and 'in vivo' in title/abstract). Despite this in vivo success in preclinical model, to date only three clinical trials are registered (source: ClinicalTrials.gov; term searched: 'electrospinning'). This is not surprising, considering that electrospinning is only 15 years old.

Overall, we believe that the full potential of imprinting and electrospinning has yet to be realized. Cellbased therapies require in vitro expansion of cells to obtain therapeutically relevant cell numbers with preserved native phenotype. Given that customarily used tissue culture plastics fail to imitate the native in vivo milieu, 2D imprinted materials with optimal/physiological surface topography and substrate rigidity are expected to be key players in the field of cell therapies to optimally propagate cells in vitro, prior to implantation. In the years to come, the manufacturing cost of $3 \mathrm{D}$ electrospun scaffolds is expected to be reduced. As such, we foresee that more and more electrospun materials will reach the clinical setting. In our view, imprinting lithography and electrospinning are due to transform healthcare.

\section{Financial \& competing interests disclosure}

This publication is supported by the Health Research Board (grant agreement number: HRA_POR/2011/84) and the Science Foundation Ireland and the European Regional Development Fund (grant agreement number: 13/RC/2073). The authors have no other relevant affiliations or financial involvement with any organization or entity with a financial interest in or financial conflict with the subject matter or materials discussed in the manuscript apart from those disclosed.

No writing assistance was utilized in the production of this manuscript.

2 Abbah S, Delgado L, Azeem A et al. Harnessing hierarchical nano- and micro-fabrication technologies for musculoskeletal tissue engineering. Adv. Healthc. Mater. 4(16), 2488-2499 (2015). 
3 Kai D, Liow S, Loh X. Biodegradable polymers for electrospinning: towards biomedical applications. Mater. Sci. Eng. C 45, 659-670 (2014).

4 Khadka D, Haynie D. Protein- and peptide-based electrospun nanofibers in medical biomaterials. Nanomedicine 8(8), 1242-1262 (2012).

5 Ingavle G, Leach J. Advancements in electrospinning of polymeric nanofibrous scaffolds for tissue engineering. Tissue Eng. B 20(4), 277-293 (2014).

6 Zheng W, Zhang W, Jiang X. Precise control of cell adhesion by combination of surface chemistry and soft lithography. Adv. Healthc. Mater. 2(1), 95-108 (2013).

7 Truskett V, Watts M. Trends in imprint lithography for biological applications. Trends Biotechnol. 24(7), 312-317 (2006).

8 Brunette D, Kenner G, Gould T. Grooved titanium surfaces orient growth and migration of cells from human gingival explants. J. Dent. Res. 62(10), 1045-1048 (1983).

9 Rajnicek A, Britland S, Mccaig C. Contact guidance of CNS neurites on grooved quartz: influence of groove dimensions, neuronal age and cell type. J. Cell Sci. 110 (23), 2905-2913 (1997).

10 Rajnicek A, Mccaig C. Guidance of CNS growth cones by substratum grooves and ridges: effects of inhibitors of the cytoskeleton, calcium channels and signal transduction pathways. J. Cell Sci. 110(23), 2915-2924 (1997).

11 Tonazzini I, Meucci S, Faraci P, Beltram F, Cecchini M. Neuronal differentiation on anisotropic substrates and the influence of nanotopographical noise on neurite contact guidance. Biomaterials 34(25), 6027-6036 (2013).

12 Mcmurray RJ, Wann AKT, Thompson CL, Connelly JT, Knight MM. Surface topography regulates WNT signaling through control of primary cilia structure in mesenchymal stem cells. Sci. Rep. 3, 3545 (2013).

13 Dalby MJ, Mccloy D, Robertson M, Wilkinson CDW, Oreffo ROC. Osteoprogenitor response to defined topographies with nanoscale depths. Biomaterials 27(8), 1306-1315 (2006).

14 Kornberg TB, Roy S. Cytonemes as specialized signaling filopodia. Development 141(4), 729-736 (2014).

15 Nikukar H, Reid S, Tsimbouri PM, Riehle MO, Curtis ASG, Dalby MJ. Osteogenesis of mesenchymal stem cells by nanoscale mechanotransduction. ACS Nano 7(3), 2758-2767 (2013).

16 Zhu J, Li J, Wang B et al. The regulation of phenotype of cultured tenocytes by microgrooved surface structure. Biomaterials 31(27), 6952-6958 (2010).

17 Dalby MJ, Gadegaard N, Tare R et al. The control of human mesenchymal cell differentiation using nanoscale symmetry and disorder. Nat. Mater. 6(12), 997-1003 (2007).

18 Unadkat H, Hulsman M, Cornelissen K et al. An algorithmbased topographical biomaterials library to instruct cell fate. Proc. Natl Acad. Sci. USA 108(40), 16565-16570 (2011).

19 Hulsman M, Hulshof F, Unadkat $\mathrm{H}$ et al. Analysis of high-throughput screening reveals the effect of surface topographies on cellular morphology. Acta Biomater. 15, 29-38 (2015).
20 Kam KR, Walsh LA, Bock SM, Ollerenshaw JD, Ross RF, Desai TA. The effect of nanotopography on modulating protein adsorption and the fibrotic response. Tissue Eng. A 20(1-2), 130-138 (2014).

21 Perez-Garnes M, Gonzalez-Garcia C, Moratal D, Rico P, Salmeron-Sanchez M. Fibronectin distribution on demixed nanoscale topographies. Int. J. Artif. Organs 34(1), 54-63 (2011).

22 Biggs MJ, Richards RG, Gadegaard N et al. Interactions with nanoscale topography: adhesion quantification and signal transduction in cells of osteogenic and multipotent lineage. $J$. Biomed. Mater. Res. A 91(1), 195-208 (2009).

23 Davis KA, Burke KA, Mather PT, Henderson JH. Dynamic cell behavior on shape memory polymer substrates. Biomaterials 32(9), 2285-2293 (2011).

24 Gong T, Zhao K, Yang G et al. The control of mesenchymal stem cell differentiation using dynamically tunable surface microgrooves. Adv. Healthc. Mater. 3(10), 1608-1619 (2014).

25 English A, Azeem A, Spanoudes K et al. Substrate topography: a valuable in vitro tool, but a clinical red herring for in vivo tenogenesis. Acta Biomater. 27 3-12 (2015).

26 Azeem A, English A, Kumar P et al. The influence of anisotropic nano- to micro-topography on in vitro and in vivo osteogenesis. Nanomedicine 10(5), 693-711 (2015).

27 Huang L, Apkarian R, Chaikof E. High-resolution analysis of engineered type I collagen nanofibers by electron microscopy. Scanning 23(6), 372-375 (2001).

28 Huang L, Nagapudi K, Apkarian R, Chaikof E. Engineered collagen-PEO nanofibers and fabrics. J. Biomater. Sci. Polym. Ed. 12(9), 979-993 (2001).

29 Zeugolis D, Khew S, Yew E et al. Electro-spinning of pure collagen nano-fibres - just an expensive way to make gelatin? Biomaterials 29(15), 2293-2305 (2008).

30 Yang L, Fitié C, Van Der Werf K, Bennink M, Dijkstra P, Feijen J. Mechanical properties of single electrospun collagen type I fibers. Biomaterials 29(8), 955-962 (2008).

31 Dang J, Leong K. Myogenic induction of aligned mesenchymal stem cell sheets by culture on thermally responsive electrospun nanofibers. Adv. Mater. 19(19), 1775-2779 (2007).

32 Huang C, Soenen S, Rejman J et al. Stimuli-responsive electrospun fibers and their applications. Chem. Soc. Rev. 40(5), 2417-2434 (2011).

33 Xu C, Inai R, Kotaki M, Ramakrishna S. Aligned biodegradable nanofibrous structure: a potential scaffold for blood vessel engineering. Biomaterials 25(5), 877-886 (2004).

34 Yang F, Murugan R, Wang S, Ramakrishna S. Electrospinning of nano/micro scale poly(L-lactic acid) aligned fibers and their potential in neural tissue engineering. Biomaterials 26(15), 2603-2610 (2005).

35 Yin Z, Chen X, Chen J et al. The regulation of tendon stem cell differentiation by the alignment of nanofibers. Biomaterials 31(8), 2163-2175 (2010).

36 Vaquette C, Cooper-White J. Increasing electrospun scaffold pore size with tailored collectors for improved cell penetration. Acta Biomater. 7(6), 2544-2557 (2011). 
37 Cheng Q, Lee B, Komvopoulos K, Li S. Engineering the microstructure of electrospun fibrous scaffolds by microtopography. Biomacromolecules 14(5), 1349-1360 (2013).

38 Fuller K, Gaspar D, Delgado L, Pandit A, Zeugolis D. The influence of porosity and pore shape on structural, mechanical and biological properties of poly $\varepsilon$-caprolactone electro-spun fibrous scaffolds. Nanomedicine (In Press).

39 Brown T, Dalton P, Hutmacher D. Direct writing by way of melt electrospinning. Adv. Mater. 23(47), 5651-5657 (2011).

40 Brown T, Slotosch A, Thibaudeau L et al. Design and fabrication of tubular scaffolds via direct writing in a melt electrospinning mode. Biointerphases 7(1-4), 13 (2012).

41 Fuller K, Pandit A, Zeugolis D. The multifaceted potential of electro-spinning in regenerative medicine. Pharm. Nanotechnol. 2(1), 23-34 (2014).

42 Choi J, Kim H, Yoo H. Electrospinning strategies of drugincorporated nanofibrous mats for wound recovery. Drug Deliv. Transl. Res. 5(2), 137-145 (2015).

43 Hu X, Liu S, Zhou G, Huang Y, Xie Z, Jing X. Electrospinning of polymeric nanofibers for drug delivery applications. J. Control. Release 185 12-21 (2014).

44 Braghirolli D, Zamboni F, Acasigua G, Pranke P. Association of electrospinning with electrospraying: a strategy to produce $3 \mathrm{D}$ scaffolds with incorporated stem cells for use in tissue engineering. Int. J. Nanomedicine 10 5159-5169 (2015).

45 Townsend-Nicholson A, Jayasinghe S. Cell electrospinning: a unique biotechnique for encapsulating living organisms for generating active biological microthreads/scaffolds. Biomacromolecules 7(12), 3364-3369 (2006).

46 Jayasinghe S, Irvine S, Mcewan J. Cell electrospinning highly concentrated cellular suspensions containing primary living organisms into cell-bearing threads and scaffolds. Nanomedicine 2(4), 555-567 (2007).
47 Stankus J, Soletti L, Fujimoto K, Hong Y, Vorp D, Wagner W. Fabrication of cell microintegrated blood vessel constructs through electrohydrodynamic atomization. Biomaterials 28(17), 2738-2746 (2007).

48 Chen Y, Zeng D, Ding L et al. Three-dimensional poly( $\varepsilon$-caprolactone) nanofibrous scaffolds directly promote the cardiomyocyte differentiation of murine-induced pluripotent stem cells through Wnt/ $\beta$-catenin signaling. BMC Cell Biol. 16, 22 (2015).

49 Cui X, Liu M, Wang J, Zhou Y, Xiang Q. Electrospun scaffold containing TGF- $\beta 1$ promotes human mesenchymal stem cell differentiation towards a nucleus pulposus-like phenotype under hypoxia. IET Nanobiotechnol. 9(2), 76-84 (2015).

50 Jiang X, Nai M, Lim C, Levisage C, Chan J, Chew S. Polysaccharide nanofibers with variable compliance for directing cell fate. J. Biomed. Mater. Res. A 103(3), 959-968 (2015).

51 Cardwell R, Dahlgren L, Goldstein A. Electrospun fibre diameter, not alignment, affects mesenchymal stem cell differentiation into the tendon/ligament lineage. J. Tissue Eng. Regen. Med. 8(12), 937-945 (2014).

52 Prabhakaran M, Venugopal J, Ramakrishna S. Mesenchymal stem cell differentiation to neuronal cells on electrospun nanofibrous substrates for nerve tissue engineering. Biomaterials 30 (28), 4996-5003 (2009).

53 Yan J, Qiang L, Gao Y et al. Effect of fiber alignment in electrospun scaffolds on keratocytes and corneal epithelial cells behavior. J. Biomed. Mater. Res. A 100 (2), 527-535 (2012).

54 Wu J, Du Y, Watkins S, Funderburgh J, Wagner W. The engineering of organized human corneal tissue through the spatial guidance of corneal stromal stem cells. Biomaterials 33(5), 1343-1352 (2012). 